BULLETIN OF THE

AMERICAN MATHEMATICAL SOCIETY

Volume 81, Number 4, July 1975

\title{
QUASI-ANALYTIC VECTORS AND QUASI-ANALYTIC FUNCTIONS
}

\author{
BY PAUL R. CHERNOFF ${ }^{1}$
}

1. Introduction. The theory of quasi-analytic classes is a part of function theory that is now over fifty years old. The notion of a quasianalytic vector is a relatively recent development in operator theory. My purpose here is to discuss the mutual interaction of these ideas, and in particular to show how the operator-theoretic point of view leads in a natural way to broad and interesting generalizations of some of the classical results.

I will begin by recalling some operator theory. Let $A$ be an operatorunbounded in general-with domain $\mathscr{D}(A)$ in a Banach space $X$. A vector $x$ is a $C^{\infty}$ vector for $A$ if $x$ belongs to $\mathscr{D}^{\infty}(A)=\bigcap_{n=1}^{\infty} \mathscr{D}\left(A^{n}\right)$. (Think of the example: $A=D=$ differentiation. Then $C^{\infty}$ vectors are just $C^{\infty}$ functions.) An analytic vector for $A$ is a $C^{\infty}$ vector $x$ such that the series $\sum_{n=0}^{\infty}\left(t^{n} / n !\right)\left\|A^{n} x\right\|$ has a positive radius of convergence. This is a growth condition on $\left\|A^{n} x\right\|$; namely, $\left\|A^{n} x\right\|^{1 / n}=O(n)$. $\mathscr{D}^{a}(A)$ will denote the space of analytic vectors for $A$.

Analytic vectors were introduced by Nelson in 1959 [15]. Among many other things, he proved the following fundamental fact.

1.1. TheOREM A. Let A be a symmetric operator on a Hilbert space $H$. If $A$ has a dense set of analytic vectors, then $A$ is essentially selfadjoint (that is, its closure is selfadjoint).

PROOF. By a well-known theorem of Naymark, there is an extension $A^{0}$ of $A$ (on a possibly larger Hilbert space $K \supseteq H$ ) which is selfadjoint. Let $U_{t}=\exp \left(i t A^{0}\right)$ be the one-parameter group generated by $A^{0}$.

To show that $A$ is essentially selfadjoint, we must prove that $A+i$ and $A-i$ have dense ranges. Suppose that $y$ is orthogonal to the range of $A+i$. Then in particular $y$ is orthogonal to $(A+i) \mathscr{D}^{a}(A)$. Then for all $x \in \mathscr{D}^{a}(A)$, $(A x, y)=-i(x, y)$. If $x \in \mathscr{D}^{a}(A)$ then $A^{n} x \in \mathscr{D}^{a}(A)$ for all positive integers $n$, and it follows that

An address delivered at the seven hundred eighteenth meeting of the American Mathematical Society in Los Angeles on November 23, 1974, by invitation of the Committee to Select Hour Speakers for Far Western Sectional Meetings.

AMS (MOS) subject classifications (1970). Primary 26A93, 30A78, 47B25, 47D05.

Key words and phrases. Analytic vector, quasi-analytic vector, quasi-analytic class, Denjoy-Carleman theorem, symmetric operator, essential selfadjointness.

1 Research partially supported by NSF Grant GP-30798X. 


$$
\left(A^{n} x, y\right)=(-i)^{n}(x, y) \text {. }
$$

Now define $f(t)=\left(U_{t} x, y\right)$; $f$ is an analytic function because $x$ is an analytic vector. Moreover, from (1),

$$
f^{(n)}(0)=\left(i^{n} A^{n} x, y\right)=(x, y)
$$

for all $n$. Conclusion: $f(t)=(x, y) e^{t}$.

But $f$ is bounded on $(-\infty, \infty)$ because $U_{t}$ is unitary. Hence $(x, y)$ must be zero. Thus $y$ is orthogonal to $\mathscr{D}^{a}(A)$, and therefore $y=0$ and $A+i$ has dense range. Similarly, $A-i$ has dense range.

A key ingredient in the above argument was the fact that two analytic functions, all of whose derivatives agree at a point, must coincide. Hadamard long ago raised the question of characterizing other classes of functions with this property. More precisely, let $I$ be an interval and $C$ a subset of $C^{\infty}(I) . C$ is said to be a quasi-analytic class provided the following condition is satisfied: if $f, g \in C$ and $x_{0} \in I$ with $D^{n} f\left(x_{0}\right)=D^{n} g\left(x_{0}\right)$ for all $n$, then $f=g$. Now, a $C^{\infty}$ function is analytic provided its successive derivatives satisfy growth restrictions in accordance with Cauchy's estimates. It is therefore reasonable to seek less restrictive growth conditions that nevertheless imply quasi-analyticity. Accordingly, given a sequence $\left\{M_{n}\right\}_{0}^{\infty}$ of nonnegative numbers, we define $C\left\{M_{n}\right\}$ to be the class of all $C^{\infty}$ functions $f$ on $I$ such that $\left\|D^{n} f\right\|_{\infty} \leqq \lambda^{n} M_{n}$ for some $\lambda$ (depending on $f) . C\left\{M_{n}\right\}$ is a linear subspace of $C^{\infty}(I)$. Hadamard's question received a nice answer in the form of the following theorem of Denjoy and Carleman. (Actually Denjoy proved a special case, and conjectured the result which was ultimately established by Carleman.)

1.2. Denjoy-Carleman theorem. $C\left\{M_{n}\right\}$ is a quasi-analytic class if and only if the least nonincreasing majorant of the series $\sum_{n=1}^{\infty} M_{n}^{-1 / n}$ diverges. (If the sequence $\left\{M_{n}\right\}_{0}^{\infty}$ is $\log$ convex, i.e. if $\log M_{n}$ is a convex function of $n$, this means that the series itself diverges.)

For a short proof of this theorem, based on ideas of Paley-Wiener, see Rudin [18, Chapter 19].

There is an interesting relation between quasi-analyticity and the question of uniqueness in the classical Hamburger moment problem. Briefly, a sequence $\left\{a_{k}\right\}_{k \geq 0}$ is of the form $a_{k}=\int_{-\infty}^{\infty} t^{k} d \mu$ for some positive measure $\mu$ on the real line-i.e. $a_{k}$ is the $k$ th moment of $\mu$-if and only if the sequence is positive definite $\left(\sum_{k, l} a_{k+l} \xi_{k} \bar{\xi}_{l} \geqq 0\right.$ for all sequences $\left\{\xi_{l}\right\}$ of complex numbers). Carleman used his theory of quasi-analytic classes to show that the measure $\mu$ is unique provided that the series $\sum_{n=1}^{\infty} a_{2 n}^{-1 / 2 n}$ diverges. We shall return to this connection in $\$ 3$.

2. Quasi-analytic vectors. In 1965 Nussbaum [15] invented the 
notion of quasi-analytic vector, guided by Nelson's work and the DenjoyCarleman theorem. If $A$ is an operator on $X$, we call a vector $x$ in $\mathscr{D}^{\infty}(A)$ quasi-analytic for $A$ provided that the least nonincreasing majorant of the series $\sum\left\|A^{n} x\right\|^{-1 / n}$ diverges. The set of these vectors is denoted by $\mathscr{D}^{a a}(A)$.

It is easy to see that analytic vectors are quasi-analytic: $\mathscr{D}^{a}(A) \subseteq \mathscr{D}^{a a}(A)$. However, $\mathscr{D}^{a a}(A)$ is not necessarily a linear space. (It is also worth noting that for symmetric operators on Hilbert space, the sequence $\left\|A^{n} x\right\|$ is $\log$ convex. This is not so in general, although there are interesting inequalities connecting the quantities $\left\|A^{n} x\right\|$; all this goes back to the classical work of Landau and Kolmogoroff [11] for the operator $d / d x$ on $L^{\infty}(\boldsymbol{R})$.)

An important observation is that if $x$ is quasi-analytic, then so is $A x$. This follows readily from a little-known inequality of Carleman [2, p. 105] which deserves to be better known: for any sequence $a_{v} \geqq 0$,

$$
\sum_{v=2}^{n} a_{v}^{1-1 / v} \leqq \sum_{v=2}^{n} a_{v}+2\left[\sum_{v=2}^{n} a_{v}\right]^{1 / 2}
$$

It follows that $\sum M_{n}^{-1 / n}$ diverges if and only if $\sum M_{n+1}^{-1 / n}$ diverges; and a similar statement holds for the least nonincreasing majorants. Now take $M_{n}=\left\|A^{n} x\right\|$ to conclude that $x \in \mathscr{D}^{a a}(A)$ implies $A x \in \mathscr{D}^{a a}(A)$.

Nussbaum generalized Nelson's theorem by proving its analogue for quasi-analytic vectors:

2.1. TheOREM QA. Let $A$ be a symmetric operator on Hilbert space $H$. Suppose that the set $\mathscr{D}^{q a}(A)$ of quasi-analytic vectors has a dense span. Then $A$ is essentially selfadjoint.

Nussbaum's original proof utilized Carleman's work on the classical moment problem. However, one can give a proof by simply mimicking our proof of Theorem A, making use of the Denjoy-Carleman theorem at the crucial point. So in a sense Theorem QA is a "corollary" of the Denjoy-Carleman theorem. One can also extend Theorem QA to semigroup generators in Banach spaces by the same technique; see [3], [8], as well as the proof of Theorem 5.3 below.

3. Some classical corollaries. It is amusing to observe that the sufficiency of the Denjoy-Carleman criterion for quasi-analyticity is actually a special case of Theorem QA. Here is a proof of an $L^{2}$ version.

3.1. THEOREM. Let $f$ be a $C^{\infty}$ function on $(-\infty, \infty)$ such that for all $n, D^{n} f$ is in $L^{2}$, and $\left\|D^{n} f\right\|_{2}=M_{n}$, where $\sum M_{n}^{-1 / n}$ diverges. Suppose that there is a point $x_{0}$ where $D^{n} f\left(x_{0}\right)=0$ for all $n$. Then $f$ vanishes identically.

Proof. We may suppose that $x_{0}=0$. It is enough to prove that $f$ 
vanishes to the right of 0 . Accordingly, consider the operator $A=i D$ on $H=L^{2}(0, \infty)$, with $\mathscr{D}(A)$ consisting of those functions $g$ in $L^{2}$ such that $g$ is absolutely continuous, $D g$ is in $L^{2}$, and $g(0)=0$. Note that $A$ is symmetric and closed, but not selfadjoint (because $e^{-x}$ is orthogonal to the range of $A-i)$. The hypothesis says that $f$ is a quasi-analytic vector for $A$.

A straightforward calculation shows that, for any real $k, e^{i k x} f(x)$ is also a quasi-analytic vector for $A$. It follows that $L^{2}$ (support $f$ ) is in the closure of the span of $\mathscr{D}^{a a}(A)$. Moreover, translates of quasi-analytic vectors are quasi-analytic for $A$. Hence unless $f$ vanishes, $\mathscr{D}^{q a}(A)$ has a dense span in $H$. But this would imply that $A$ is selfadjoint, a contradiction.

The $L^{\infty}$ version of the Denjoy-Carleman theorem follows as an easy corollary. By using the $\left(C_{0}\right)$ semigroup version of Theorem QA to which we have already alluded, the same argument applies to the other $L^{p}$ norms, $1 \leqq p<\infty$.

An important observation is that the general argument used above applies to many differential operators besides $D$, and in this way we are led to new examples of quasi-analytic classes. Rather than attempting to give the most general possible results along these lines, we shall illustrate the method with a specific case below, in $\S 6$. Related work, but using quite different methods, has been carried out by a number of authors [1], [4], [10].

We can also use Theorem QA to demonstrate Carleman's uniqueness condition for the Hamburger moment problem, mentioned at the end of $\S 1$.

Thus, let $\left\{a_{k}\right\}_{0}^{\infty}$ be the moment sequence of a measure $\mu$ on $(-\infty, \infty)$ : $a_{k}=\int_{-\infty}^{\infty} t^{k} d \mu(t)$. Assuming that $\sum a_{2 n}^{-1 / 2 n}=\infty$, we shall prove that $\mu$ is uniquely determined.

For this, consider the selfadjoint operator $A=$ multiplication by $t$ on $L^{2}(R, \mu)=H$. Let $u$ be the constant function $u(t)=1$. Note that $u \in \mathscr{D}^{\infty}(A)$. Also, $a_{n}=\left(A^{n} u, u\right)$, so $a_{2 n}=\left(A^{2 n}, u, u\right)=\left\|A^{n} u\right\|^{2}$. Thus Carleman's condition says that $\sum\left\|A^{n} u\right\|^{-1 / n}=\infty$, that is, that $u$ is a quasianalytic vector for $A$.

Then $t^{n}=A^{n} u$ is also quasi-analytic. It follows that the restriction $A_{1}$ of $A$ to $\mathscr{P}$ (the polynomials in $t$, i.e., the span of $u, A u, A^{2} u, \cdots$ ) is essentially selfadjoint in $H_{1}$, the closure of $\mathscr{P}$ in $H$. It follows that $H_{1}$ is invariant under the one-parameter group $e^{i s A}$. We can now conclude that $H_{1}=H$, i.e., that $\mathscr{P}$ is dense in $L^{2}(R, \mu)$. Indeed, suppose that $y \in \mathscr{P} \perp$. Then for all $s$,

$$
0=\left(y, e^{i s A} u\right)=\int e^{-i s t} y(t) d \mu(t)
$$


Conclusion: $y(t)=0 \mu$-a.e.

Now if $\nu$ is another measure generating the moment sequence $\left\{a_{k}\right\}_{0}^{\infty}$, it follows that the identity on polynomials $\mathscr{P}$ extends to an isometry of $L^{2}(R, \mu)$ with $L^{2}(R, \nu)$, whence $\nu=\mu$.

4. Semianalytic and Stieltjes vectors. We say that a vector $x \in$ $\mathscr{D}^{\infty}(A)$ is a Stieltjes vector for $A$ if the least nonincreasing majorant of the series $\sum\left\|A^{n} x\right\|^{-1 / 2 n}$ diverges. Stieltjes vectors were invented by Nussbaum [17] and, independently, by Masson and McClary [14], to whom the terminology is due. (The term "Stieltjes vector" was suggested by the Stieltjes moment problem, which is the analogue for the half-line of the Hamburger moment problem.) In [14], the theory of Stieltjes vectors is applied to prove essential selfadjointness of certain Hamiltonian operators assisting in quantum field theory. The fundamental theorem is

4.1. Theorem S. Let A be a semibounded symmetric operator on Hilbert space $H$. Suppose that the set of Stieltjes vectors $\mathscr{D}^{s}(A)$ has dense span. Then $A$ is essentially selfadjoint.

Note that $\mathscr{D}^{s}(A) \supseteq \mathscr{D}^{a a}(A)$, so Theorem $\mathrm{S}$ is a strengthening of Theorem QA for a more restricted class of operators $A$.

In a paper [19] which presented a simplified proof of Theorem 5, Simon introduced the related idea of semianalytic vectors, which are to Stieltjes vectors what analytic vectors are to quasi-analytic vectors. Namely a vector $x$ is semianalytic for $A$ if the series $\sum_{n=0}^{\infty}\left(t^{n} /(2 n) !\right)\left\|A^{n} x\right\|$ has a positive radius of convergence. We have the corresponding theorem (actually a special case of Theorem $S$ ).

4.2. TheOREM SA. Let $A$ be a semibounded symmetric operation on Hilbert space $H$. Suppose that the set of semianalytic vectors $\mathscr{D}^{s a}(A)$ is dense. Then $A$ is essentially selfadjoint.

The original proofs of these theorems relied on moment problem techniques. However, they are actually corollaries of Theorems QA and A respectively, via an operator-theoretic technique. The basic idea is quite simple (for details, see [3]). Without loss of generality, we may assume $A \geqq I$. Consider the operator

$$
B=i\left[\begin{array}{cc}
0 & I \\
-A & 0
\end{array}\right]
$$

on the Hilbert space $K=H_{1} \oplus H$. Here $H_{1}$ is the domain of the square root of the Friedrichs extension of $A$; equivalently, $H_{1}$ is the completion of $\mathscr{D}(A)$ in the norm $\|x\|_{1}^{\varepsilon}=(A x, x)$. It is straightforward to verify that $B$ is symmetric, and $B$ is essentially selfadjoint if and only if $A$ is. Moreover we can manufacture a supply of analytic (respectively, quasi-analytic) 
vectors for $B$ from semianalytic (respectively, Stieltjes) vectors for $A$, and in this way we draw the desired conclusions about essential selfadjointness.

N.B. One might at first think that the preceding "doubling" technique could be iterated to prove a "hemi-semi-analytic vector" theorem. However, this is not so, because the operator $B$ is no longer semibounded.

Carleman's sufficient condition for uniqueness in the Stieltjes moment problem follows from Theorem S. Suppose that $\mu$ is a measure on $[0, \infty)$ generating the moment sequence $\left\{c_{n}\right\}_{0}^{\infty}: c_{n}=\int_{0}^{\infty} t^{n} d \mu$. If $\sum c_{n}^{-1 / 2 n}=\infty$ then $\mu$ is uniquely determined. This can be proved by applying Theorem $\mathrm{S}$ to the operator $A$ of multiplication by $t$ on $L^{2}(0, \infty)$. (Note that $A$ is semibounded.) The argument is quite similar to the derivation of the analogous result for the Hamburger moment problem.

5. Stieltjes vectors and boundary values of holomorphic functions. We shall apply Theorem $S$ to deduce the following variant of a theorem of Korenbljum ([12]; also cf. [5], [6]), which describes quasi-analytic classes within the class of boundary values of functions holomorphic in a halfplane.

5.1. THEOREM. Let $f$ belong to $H^{2}(U)$, where $U$ is the upper half-plane. Assume that:

(i) for all $n, f^{(n)}(z) \in H^{2}(U)$;

(ii) for all $n, f^{(n)}(0)=0$;

(iii) $\left\|f^{(n)}\right\|_{2} \leqq M_{n}$, with $\sum M_{n}^{-1 / 2 n}=\infty$.

Then $f$ is identically 0.

Proof. $H^{2}(U)$ is unitarily equivalent to $L^{2}(0, \infty)$ via the Fourier transform. On $L^{2}(0, \infty)$ define an operator $A$ by $A h(t)=t h(t)$, with dense domain

$$
\mathscr{D}(A)=\left\{h \in L^{2}: \operatorname{th}(t) \in L^{2} \quad \text { and } \int_{0}^{\infty} h(t) d t=0\right\} .
$$

It is clear that $A$ is symmetric and positive. But $A$ is not essentially selfadjoint; indeed, $k(t)=(t-i)^{-1}$ is obviously orthogonal to the range of $A+i$.

Now let $f$ satisfy the hypotheses of the theorem. Let $g \in L^{2}(0, \infty)$ be the Fourier transform of $f$. Hypothesis (i) implies that for all $n, t^{n} g(t) \in$ $L^{2}(0, \infty)$, while (ii) implies that $\int_{0}^{\infty} t^{n} g(t) d t=0$. In other words, $g$ is a $C^{\infty}$ vector for $A$. Finally, since $\left\|A^{n} g\right\|=\left\|f^{(n)}\right\|$, (iii) says that $g$ is a Stieltjes vector for $A$.

Consider $\mathscr{S}$, the set of all Stieltjes vectors for $A$. It is easy to see that $\mathscr{S}$ is closed under right translations. Likewise, $\mathscr{S}$ is closed under dilation: if $g \in \mathscr{S}$ and $\alpha>0$, the function $g(\alpha t)$ is in $\mathscr{S}$. Accordingly, applying the 
Fourier transform, $\hat{\mathscr{S}} \subseteq H^{2}(U)$ is invariant under multiplication by all functions $e^{i \lambda z}$ for $\lambda \geqq 0$, as well as invariant under dilations.

The closed linear span $M$ of $\mathscr{S}$ is thus an "invariant subspace" of $H^{2}(U)$ which is dilation invariant as well. The well-known structure of invariant subspaces [18] (either $M=0$ or $M=q \cdot H^{2}(U)$ for an essentially unique "inner function" $q$ ) implies that $M=(0)$ or $H^{2}(U)$ (for $q$ must be dilation invariant, hence constant).

But if $f \neq 0$ then $g \neq 0$ so $M=H^{2}(U)$; that is, $\mathscr{S}=\mathscr{D}^{s}(A)$ has dense span, so $A$ is essentially selfadjoint by Theorem $\mathrm{S}$. This is a contradiction.

5.2. CoRollary. In the theorem, replace $H^{2}(U)$ by $H^{\infty}(U)$, and the $L^{2}$ norms by sup norms. The conclusion remains the same.

Proof. Apply the previous theorem to $f(z) /(z+i)$.

We can go on to draw operator conclusions from this functiontheoretic fact; specifically, we can generalize Theorem $\mathrm{S}$ from semibounded selfadjoint operators to the broader context of generators of holomorphic semigroups.

5.3. TheOREM. Let $A$ be an operator on a Banach space $X$. Suppose that $A$ has an extension $A^{0}$ which generates a semigroup $\exp \left(t A^{0}\right)$, uniformly bounded in norm for $\operatorname{Re} t \geqq 0$ and holomorphic for $\operatorname{Re} t>0$. Assume that the set $\mathscr{D}^{s}(A)$ of Stieltjes vectors of a has dense span. Then $A^{0}=\bar{A}$, the closure of $A$.

Proof. By the Hille-Yosida theorem [9], it is enough to show that the range of $I-A$ is dense. Suppose that $\varphi \in X^{*}$ annihilates this range. Then, for all $x \in \mathscr{D}(A)$,

$$
\langle\varphi, A x\rangle=\langle\varphi, x\rangle \text {. }
$$

Now suppose that $x \in \mathscr{D}^{8}(A)$. Consider the function $f(t)=\left\langle\varphi, \exp \left(t A^{0}\right) x\right\rangle$; $f$ is $C^{\infty}$ for $\operatorname{Re} t \geqq 0$, holomorphic for $\operatorname{Re} t>0$, and all derivatives $f^{(n)}$ are uniformly bounded. Moreover, by induction on $n, f^{(n)}(0)=\left\langle\varphi, A^{n} x\right\rangle=$ $\langle\varphi, x\rangle$.

Now consider the function $h(t)=e^{-t} f(t)-\langle\varphi, x\rangle$. The function $h$ is $C^{\infty}$ for $\operatorname{Re} t \geqq 0$, holomorphic for $\operatorname{Re} t>0$, and one can check that $h^{(n)}(0)=$ 0 for all $n$; moreover,

$$
\left\|D^{n} h\right\|_{\infty} \leqq \sum_{r=0}^{n}\left(\begin{array}{l}
n \\
r
\end{array}\right)\left\|D^{r} f\right\|_{\infty} .
$$

Now, by work of Kolmogoroff [11] (cf. [13, p. 216]) we have

$$
\left\|D^{r} f\right\|_{\infty} \leqq 2\|f\|_{\infty}^{1-r / n}\left\|D^{n} f\right\|_{\infty}^{r / n}, \quad 0 \leqq r \leqq n .
$$

Hence, for some constants $C, C^{\prime}$, etc., 


$$
\begin{aligned}
\left\|D^{n} h\right\|_{\infty} & \leqq C \sum_{r=0}^{n}\left(\begin{array}{l}
n \\
r
\end{array}\right)\left\|D^{n} f\right\|_{\infty}^{r / n}=C\left(1+\left\|D^{n} f\right\|_{\infty}^{1 / n}\right)^{n} \\
& \leqq C^{\prime}\left\|D^{n} f\right\|_{\infty} \quad \text { for } n \text { large. }
\end{aligned}
$$

But $\left\|D^{n} f\right\|_{\infty}=\sup \left|\left\langle\varphi, \exp \left(t A^{0}\right) A^{n} x\right\rangle\right| \leqq C^{\prime \prime}\left\|A^{n} x\right\|$. Since $x$ is a Stieltjes vector, it follows that the series $\sum_{n=1}^{\infty}\left\|D^{n} h\right\|_{\infty}^{-1 / 2 n}$ diverges. Conclusion: $h=0$, by 5.2 .

Thus $f(t)=\langle\varphi, x\rangle e^{t}$; that is,

$$
\left\langle\left(\exp \left(t A^{0}\right)\right)^{*} \varphi, x\right\rangle=\left\langle e^{t} \varphi, x\right\rangle
$$

for all $x \in \mathscr{D}^{s}(A)$. Since $\mathscr{D}^{s}(A)$ has dense span, we deduce that $\left(\exp \left(t A^{0}\right)\right)^{*} \varphi=e^{t} \varphi$. But $\left\|\left(\exp \left(t A^{0}\right)\right)^{*} \varphi\right\|$ is uniformly bounded for $\operatorname{Re} t \geqq 0$, and so we must have $\varphi=0$. Thus $I-A$ has dense range.

An unsatisfactory feature of Theorem 5.3 is the a priori assumption that the extension $A^{0}$ exists. (In the Hilbert space context of Theorem $\mathrm{S}$ this was automatic because of the availability of the Friedrichs extension.) Actually, it is not hard to see that we need only the existence of a suitable generator $A^{0}$ extending $A$ on a Banach space $Y$ perhaps properly containing $X$ as a closed subspace. (An analogous situation is discussed in [3, §3].) It would be interesting to determine the conditions under which such extensions exist.

Finally, we mention a result of Korenbljum's [12] which generalizes Theorem 5.1. Let $S_{\alpha}$ be a closed sector in the complex plane: $S_{\alpha}=$ $\{z:|\arg z| \leqq \alpha \pi\}$. Suppose that $f$ is $C^{\infty}$ in $S_{\alpha}$, holomorphic in the interior of $S_{\alpha}$, and $\left\|f^{(n)}\right\|_{\infty} \leqq M_{n}$. Suppose also that $f^{(n)}(0)=0$ for all $n$. Then if the series $\sum_{n=1}^{\infty} M_{n}^{-1 /(\alpha+1) n}$ diverges, the function $f$ is identically zero. (If $\alpha=0$ this reduces to the Denjoy-Carleman theorem, while if $\alpha=1$ we get Theorem 5.1 and its corollary.) Can this be deduced by operatortheoretic methods from Theorem QA as we deduced Theorem 5.1? In any case, Korenbljum's theorem can be applied to semigroups holomorphic in $S_{\alpha}$, yielding a result analogous to Theorem 5.3.

6. Quasi-analytic classes and partial differential operators. The methods employed in $\$ 3$ in connection with the Denjoy-Carleman theorem can be applied to many ordinary and partial differential operators to generate quasi-analytic classes. The Laplacian $\Delta$ in $\boldsymbol{R}^{d}$ provides a very nice illustration.

6.1. Theorem. Let $f$ be a $C^{\infty}$ function on $\boldsymbol{R}^{d}$. Assume that, for all $n$, $\Delta^{n} f$ is in $L^{2}$, and that $\sum_{n=1}^{\infty}\left\|\Delta^{n} f\right\|^{-1 / 2 n}=\infty$. Suppose that all partial derivatives of $f$ vanish at 0 . Then $f$ is identically zero.

PRoof. First suppose that the dimension $d \leqq 3$. Let $A$ be the operator $-\Delta$ restricted to the domain $\mathscr{D}(A)$ of $C^{\infty}$ functions such that all deriva- 
tives lie in $L^{2}$ and all vanish at the origin. $\mathscr{D}(A)$ is of course dense, and $A$ is symmetric and semibounded. But $A$ is not essentially selfadjoint; e.g., one can verify that $(1-\Delta) \mathscr{D}(A)$ is not dense in $L^{2}$ by Fourier transform techniques.

The hypothesis says that $f$ is a Stieltjes vector for $A$. It is easy to check that, for all $\alpha \in \boldsymbol{R}^{d}, e^{i \alpha \cdot x} f(x)$ is also a Stieltjes vector, so that $L^{2}$ (support $f$ ) is contained in the closed span of $\mathscr{D}^{s}(A)$. Moreover, $\mathscr{D}^{s}(A)$ is clearly invariant under rotations and dilations. Hence, if $f \neq 0, \mathscr{D}^{s}(A)$ has dense span. But this contradicts the fact that $A$ is not essentially selfadjoint.

In dimensions $d$ higher than 3, we have to modify the preceding argument slightly, by working on a suitable Sobolev space $H^{s}\left(\boldsymbol{R}^{d}\right)$ instead of $L^{2}\left(\boldsymbol{R}^{d}\right)=H^{0}\left(\boldsymbol{R}^{d}\right)$. Choose $s$ so that $d-3 \leqq 2 s \leqq d$. Since $d \geqq 2 s$ it follows that $\mathscr{D}(A)$ as defined above is dense in $H^{s}$; while since $d-3 \leqq 2 s$ it follows that $(1-\Delta) \mathscr{D}(A)$ is not dense, so $A$ is symmetric and semibounded but not essentially selfadjoint. Moreover by virtue of the expression of the $H^{s}$ norm in terms of the $L^{2}$ norm of a suitable power of the Laplacian, the hypothesis implies that $f$ is a Stieltjes vector for $A$ in $H^{s}$. The rest of the argument goes through as before, with minor technical changes.

6.2. Corollary Let $\left\{M_{n}\right\}_{0}^{\infty}$ be a log convex sequence. Define $\mathscr{C}\left(\left\{M_{n}\right\}, \Delta, \boldsymbol{R}^{d}\right)$ to be the class of all $C^{\infty}$ functions $f$ on $\boldsymbol{R}^{d}$ such that $\Delta^{n} f \in L^{2}$ for all $n$ and $\left\|\Delta^{n} f\right\|_{2} \leqq M_{n} \lambda^{n}$ for some constant $\lambda$. Suppose that $\sum_{n=1}^{\infty} M_{n}^{-1 / 2 n}$ diverges. Then this class is quasi-analytic.

This result is a significant strengthening of a theorem of Bochner and Taylor [1, Theorem 9]. They require $\sum_{n=1}^{\infty} M_{n}^{-1 / n}$ to be divergent. More importantly, they conclude that a function $f$ as in the hypothesis of 6.1 vanishes only under the stronger assumption that all powers of the Laplacian $\Delta^{n} f(x)$ vanish for all points $x$ in a "determining set" $U$ (i.e., a set $U \subseteq \boldsymbol{R}^{d}$ and that an analytic function which vanishes on $U$ must vanish identically). We require that all partials $D^{\alpha} f$ vanish only at a single point. (N.B. The example of $f(x)=x \exp \left(-x^{2} / 2\right)$ in one dimension shows that we must require that all partials vanish at 0 , not merely the iterates of $\Delta$, in order to conclude that $f=0$.)

It is clear that the Laplacian could be replaced by any of a wide variety of elliptic operators.

\section{REFERENCES}

1. S. Bochner and A. E. Taylor, Some theorems on quasi-analyticity for functions of several variables, Amer. J. Math. 61 (1939), 303-329.

2. T. Carleman, Les fonctions quasi-analytiques Gauthier-Villars, Paris, 1926.

3. P. R. Chernoff, Some remarks on quasi-analytic vectors, Trans. Amer. Math. Soc. 167 (1972), 105-113. MR 45 \#4193. 
4. L. Ehrenpreis, Fourier analysis in several complex variables, Pure and Appl. Math., vol. 17, Wiley, New York, 1970. MR 44 \#3066.

5. A. Gorny, Contribution à l'étude des fonctions dérivables d'une variable réelle, Acta Math. 71 (1939), 317-358. MR 1, 137; 400.

6. W. Groening, Analytic functions with quasi-analytic boundary values, Trans. Amer. Math. Soc. 143 (1969), 95-107.

7. - Quasi-analyticity for functions of several variables, Duke Math. J. 38 (1971), 109-115. MR 42 \#7933.

8. M. Hasegawa, On quasi-analytic vectors for dissipative operators, Proc. Amer. Math. Soc. 29 (1971), 81-84. MR 43 \#981.

9. E. Hille and R. S. Phillips, Functional analysis and semi-groups, rev. ed., Amer. Math. Soc. Colloq. Publ., vol. 31, Amer. Math. Soc., Providence, R.I., 1957. MR 19, 664.

10. V. G. Hryptun, Classes of functions quasianalytic with respect to an ordinary linear differential operator, and their applications, Dokl. Akad. Nauk SSSR 177 (1967), 528-530=Soviet Math. Dokl. 8 (1967), 1456-1459. MR 37 \#2927.

11. A. N. Kolmogorov, On inequalities between the upper bounds of the successive derivatives of an arbitrary function on an infinite interval, Učen. Zap. Moskov. Gos. Univ. Mat. 30 (1939), 3-13; English transl., Amer. Math. Soc. Transl. (1) 2 (1962), 233243. MR 1, 298.

12. B. I. Korenbljum, Conditions of nontriviality of certain classes of functions analytic in a sector, and problems of quasianalyticity, Dokl. Akad. Nauk. SSSR 166 (1966), 1046-1049=Soviet Math. Dokl. 7 (1966), 232-236. MR 34 \#1528.

13. S. Mandelbrojt, Séries adherentes, régularisation des suites, applications, GauthierVillars, Paris, 1952. MR 14, 542.

14. D. Masson and $W$. McClary, Classes of $C^{\infty}$ vectors and essential self-adjointness, J. Functional Analysis 10 (1972), 19-32.

15. E. Nelson, Analytic vectors, Ann. of Math. (2) 70 (1959), 572-615. MR 21 \#5901.

16. A. E. Nussbaum, Quasi-analytic vectors, Ark. Mat. 6 (1965), 179-191. MR 33 \#3105.

17. - A note on quasi-analytic vectors, Studia Math. 33 (1969), 305-309. MR 40 \#4781.

18. W. Rudin, Real and complex analysis, McGraw-Hill, New York, 1966. MR 35 $\# 1420$.

19. B. Simon, The theory of semi-analytic vectors: A new proof of a theorem of Masson and McClary, Indiana Univ. Math. J. 20 (1970/71), 1145-1151. MR 44 \#7357. 